Journal of Engineering and Applied Sciences 15 (6): 1421-1425, 2020

ISSN: 1816-949X

(C) Medwell Journals, 2020

\title{
AHP-COA Combined Algorithm for Selecting a Digital Production Machine Design
}

\author{
${ }^{1}$ Shiva Shokoohi Mehran, ${ }^{2}$ Sanaz Vessali, ${ }^{3}$ Majid Ahmadi and ${ }^{2}$ Elham Shadkam \\ ${ }^{1}$ Department of Industrial Engineering, Faculty of Engineering, \\ Khayyam University, Mashhad, Iran \\ ${ }^{2}$ La Trobe University MEcon, MAccFin, Australia \\ ${ }^{3}$ Department of Accounting and Management, Islamic Azad University, Firoozkuh Branch, \\ Firoozkuh, Iran
}

\begin{abstract}
This study shows a COA algorithm approach in order to find Pareto solutions for multi-objective selection of advanced machining scheme. For multi-purpose evaluation in this approach, the proposed method for focusing and determining the weight value of the evaluation indices enables a Analytical Hierarchical Process (AHP) to enable the engineer to determine a variety of requirements. There are three reverse objectives: cost, quality and operation time, which are built at the same time. A pilot program has been created and its results analyzed. This optimization of the results shows that the algorithm is valid.
\end{abstract}

Key words: Pareto, requirements, optimization, multi-objective, valid, evaluation

\section{INTRODUCTION}

In the field of decision making where the choice of one of the available solutions or the prioritization of solutions has been discussed, it has been several years that decision making methods with multiple MADM indices have emerged. Among these, the hierarchical analysis method (AHP) has been used in management science more than any other method. A number of algorithms have been introduced for multi-objective optimization problems: such as Ant Colony algorithm, COA (Cuckoo Algorithm, Genetic algorithm, particle swarm optimization algorithm, fuzzy optimization algorithm, artificial lattice, etc.), COA algorithm as a global search technology is a particularly suitable method to obtain a global optimal solution to the multi-objective optimization problem because COA has advantages such as excellent flexibility, ease of operation, easy operation and short computational time. Plenty of attention has been gathering around the information production models enabled by the modern Information and Communication Technologies (ICT) and brought to the forefront by collaborative projects such as the Free/Open Source Software (FOSS) movement or the free encyclopedia Wikipedia. On the other hand, researchers such as Webster (2002a, b) have argued against the idea of an 'information society'. They emphasize the continuities of the current age with former capitalist-oriented social and economic arrangements (Webster, 2002a, b). Kumar maintains that the information explosion 'has not produced a radical shift in the way industrial societies are organized' to conclude that 'the imperatives of profit, power and control seem as predominant now as they have ever been in the history of capitalist industrialism'. The widespread adoption of ICT cannot automatically produce a better world for humanity: some technologies need the appropriate social environments to be structured in a certain way (Langdon, 1986). For instance that could be one reason why in the past decades attempts for more autonomous forms of production based on novel technologies from the 'small-is-beautiful-experiments' (Langdon, 1986) to the development of wind power from below in the 1970s proved unsuccessful. The case of the RepRap-based, Lego-built 3D printing-milling machine, with regard to Winner's and Glover's concerns, attempts to show that new means of production such as the ICT and the emerging digital fabrication capabilities, could create the appropriate knowledge-based social environments and make possible not only the independent production of information but also the independent production of modular hardware, even in such an infancy form. Blochliger and Zufferey (2008) proposed an approach called FOOPARTIALCOL which is based on Tabu search. The method considers feasible but partial solutions and tries to increase the size of the current partial solution. Ray et al. (2010) proposed an evolutionary algorithm (GA) with double point guided mutation for the graph coloring problem which could advance the performance level of simple GA dramatically. Lu and Hao (2010) present a Memetic Algorithm (MACOL) to solve the graph coloring problem. The proposed MACOL algorithm integrates several distinguished features such as Adaptive Multi-Parent Crossover (AMPaX) operator and a 
distanceand-quality based replacement criterion for pool updating. Ge et al. (2010) proposed a hybrid Chaotic Ant Swarm approach for the graph Coloring problem (CASCOL). This approach was based on a novel swarm intelligence technique called Chaotic Ant Swarm (CAS) and a simple greedy sequential coloring, First-Fit algorithm. This study shows a COA algorithm approach in order to find Pareto solutions for multi-objective selection of advanced machining scheme. For multi-purpose evaluation in this approach, the proposed method for focusing and determining the weight value of the evaluation indices enables a Analytical Hierarchical Process (AHP) to enable the engineer to determine a variety of requirements. There are three reverse objectives: cost, quality and operation time which are built at the same time. A pilot program has been created and its results analyzed. This optimization of the results shows that the algorithm is valid.

\section{MATERIALS AND METHODS}

Many or even more real engineering problems in a multi-objective optimization, maximizing performance increasing reliability, reducing costs, etc. but in conventional processing, the choice of machining plan typically only considers one specific evaluation factor. Takes. There are many factors that can influence the choice of processing method such as processing quality, processing costs, processing time, at the same time. In addition to selection one can be influenced by the assorted state and quality of assembly assets, production compatibility, managerial talent and so on. Designing is an implementation of the multi-objective machining problem that is the subject of choice. Here, designing the angular machining is essentially, under constant assembly operations and limited assembly property. The method of choice is illustrated in Fig. 1.

The AHP method determines the qualitative weight by creating multilevel decision structures and matrix pairing comparisons. In this research, there are three levels in the structure of different levels of the evaluation model, level A is the target level of machining plan, level $\mathrm{B}$ and level D components. The following are significant evaluations and evaluation variables. Cost checking, processing time, quality checking are real components of the evaluation. Processing costs consist of variable costs and fixed costs. Fixed costs are costs that do not change in a business in the applicable period or scale of production such as cost tool set, workshop assembly cost, management cost, unit cost of unit operation, technological equipment, etc. That can be selected at the same workshop or short distance between the region of those production resources, so, shipping time and cost are not taken into account. Therefore, the evaluation variables include the processing cost including the useful general

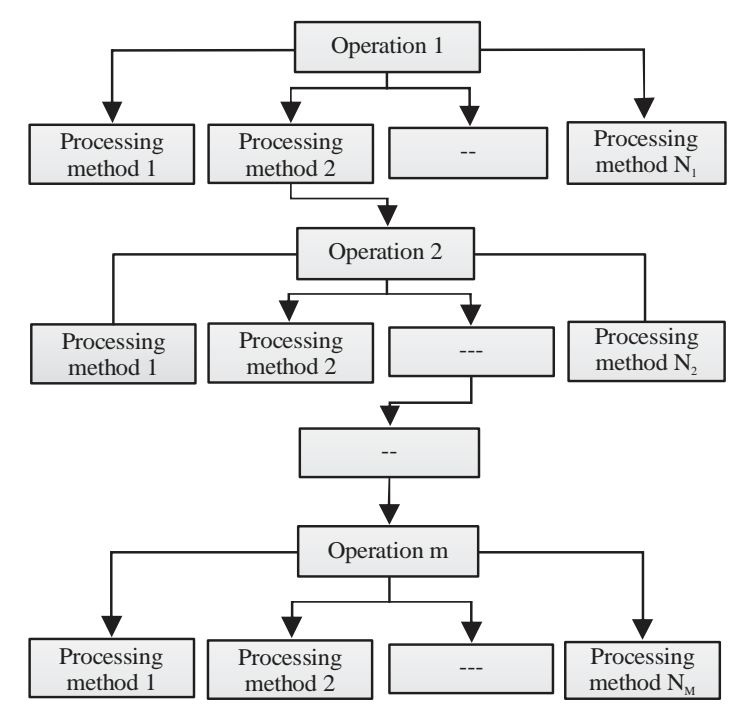

Fig. 1: Process selection chart

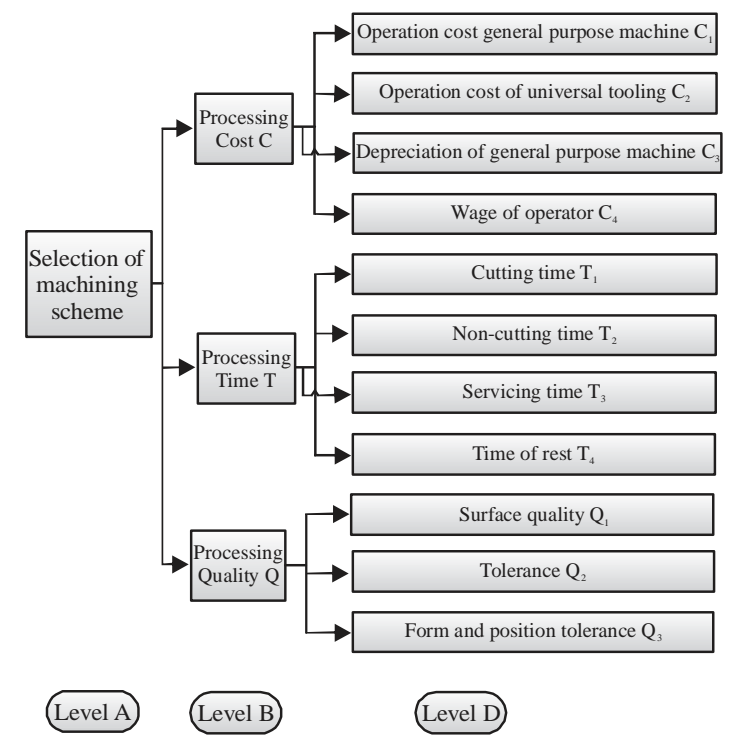

Fig. 2: Hierarchical structure for model evaluation

cost of the machine, the operating cost of the equipment, the global useful machine depreciation. The hierarchical structure for model evaluation is shown in Fig. 2.

Analytical Hierarchy Process (AHP): It has been several years that decision-making methods with multiple MADM indices have emerged in decision-making science where choosing a solution from existing solutions or prioritizing solutions. The AHP method is among the most widely used in management science. The hierarchical analysis process is one of the most popular multipurpose decision making techniques first invented by Thomas L. Sa'ati in the 1790s. The hierarchical analysis process reflects natural behavior and human thinking 
which examines complex issues based on their interactions and makes them simple to solve. The hierarchical analysis process can be used when the decision-making process has several competing options and decision criteria. The criteria can be quantitative and qualitative. The basis of this decision-making method lies in pairwise comparisons. The decision maker begins by making the decision tree hierarchical. The decision hierarchy tree shows the comparative factors and competing options evaluated in the decision. Then a series of pairwise comparisons is made. These comparisons show the weight of each factor in relation to the competing options evaluated in the decision. Finally, the logic of the hierarchical analysis process combines matrices from paired comparisons to make the optimal decision.

Principles of the analytical hierarchy process: The founder of this method (Thomas Hourly) has outlined the following four principles as the principles of the hierarchical analysis process and has based all calculations, rules and regulations on them.

Reverse condition: If element A's preference for element $B$ is n, element B's preference for element A will be $1 / n$.

Homogeneity principle: Element A must be homogenous and comparable to element $\mathrm{B}$. In other words, the superiority of element A over element B cannot be infinite or zero.

Dependency: Each hierarchical element can depend on its higher level element and this dependence can continue linearly to the highest level.

In this step, the problem and purpose of decision making are presented as hierarchical elements of decision making that are related. Decision elements include "decision indicators" and "decision options". The hierarchical analysis process requires breaking a problem with several indicators into a hierarchy of levels. The high level represents the main purpose of the decision-making process. The second level represents the major and basic indicators which may be broken down into the subsidiary and the more detailed ones at the next level. The last level presents the decision options.

\section{RESULTS AND DISCUSSION}

In Fig. 3, the Cuckoo optimization algorithm is plotted. Like other evolutionary COA algorithms, it starts with an initial population. A population of cuckoos, this population of eggs that will lay them in a host of birds. Other eggs are identified and killed by the host bird. The amount of eggs hatched indicates the suitability of the locusts in the area. The more eggs in a given area are able to survive and survive, the greater the benefit to

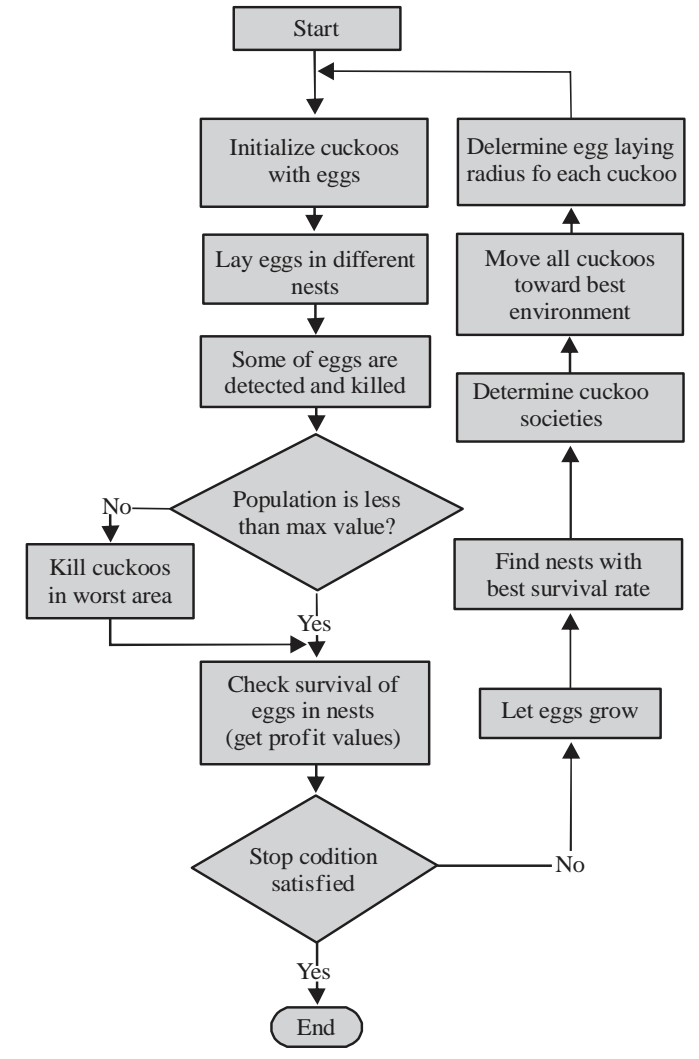

Fig. 3: Flowchart of cuckoo optimization algorithm (Rajabioun, 2011)

the area. Therefore, the situation where most eggs are saved will be the parameter that the COA intends to optimize.

Cuckoo's are looking for the best area to maximize their egg rescue. Once the chickens hatch and become mature cats, they form communities and groups. Each group has its own area of residence. The best place to live for all cuckoo. All groups will be the next destination for all cuckoo in other groups. All groups are migrating to the best available region. Each group resides in a region close to its current best position. The number of eggs that each cuckoo will lay as well as the distance of the cuckoo from the current optimal area to settle a number of oviposition radii are calculated and formed. The cuckoo then begins to randomly spawn in the lobes within their spawning radius. This stroll continues until it reaches the best location for laying the most profitable area.

Calculations of pairwise comparisons of criteria and options versus criteria are described in the article (Guan et al. 2009). In this study, the final table of pairwise comparisons of options to criteria is given in Table 1. In the strategy of the proposed COA-AHP algorithm, we try to investigate the open axis and surface roughness. Accuracy and roughness are respectively IT6 


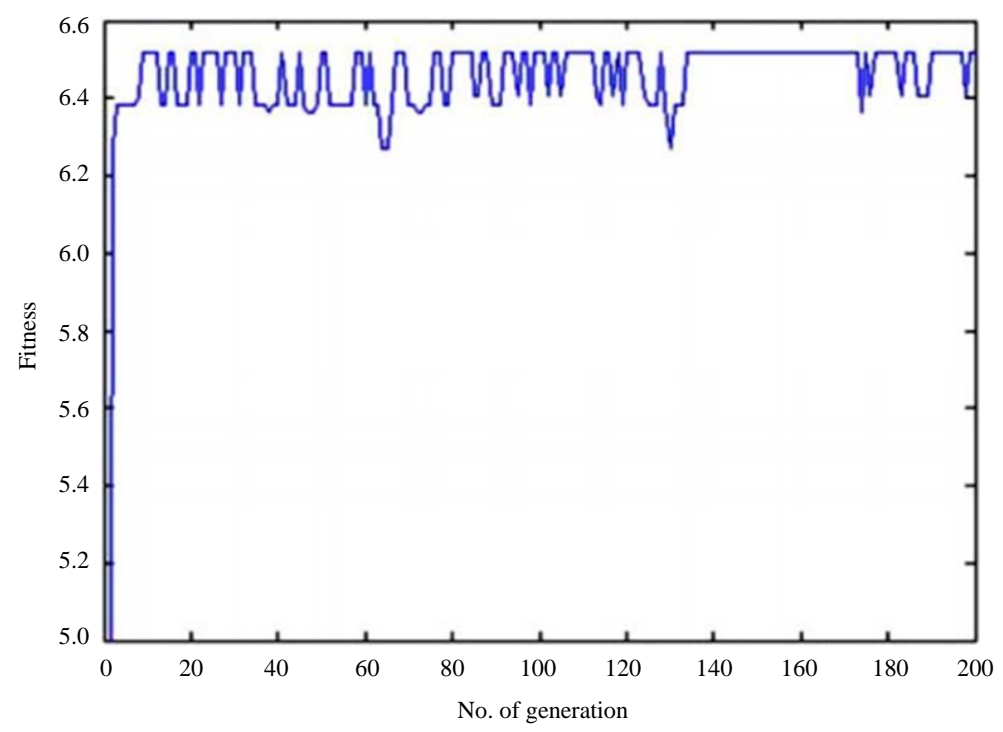

Fig. 4: GA-AHP

Table 1: Ultimate weight

\begin{tabular}{|c|c|c|c|c|}
\hline \multirow[t]{3}{*}{ B-D } & \multicolumn{3}{|l|}{$A-B$} & \multirow[t]{3}{*}{ OVERALL weight vector W } \\
\hline & B1 & B2. & B3 & \\
\hline & $0 / 4023$ & $0 / 3289$ & $0 / 2688$ & \\
\hline DI & & & & $0 / 0915$ \\
\hline D2 & & & & $0 / 0603$ \\
\hline D3 & & & & $0 / 1123$ \\
\hline D4 & & & & $0 / 1382$ \\
\hline D5 & & & & $0 / 1805$ \\
\hline D6 & & & & $0 / 0635$ \\
\hline D7 & & & & 0.0474 \\
\hline D8 & & & & 0.0375 \\
\hline D9 & & & & $0 / 1018$ \\
\hline D10 & & & & 00835 \\
\hline DII & & & & 0.0835 \\
\hline
\end{tabular}

Table 2: Information processing approach

\begin{tabular}{|c|c|c|c|c|c|c|c|c|c|c|c|c|}
\hline \multirow{6}{*}{ Face Processing } & 1.rough milling & 1 & 1 & 1 & 1 & 1 & 1 & 1 & 1 & 1 & 1 & 1 \\
\hline & 2.Foreplane & 0.6 & 0.5 & 1.2 & 1 & 0.5 & 1 & 1 & 1 & 1 & 1 & 1 \\
\hline & 1.Semi-Finish milling & 1 & 1 & 1 & 1 & 1 & 1 & 1 & 1 & 1 & 1 & 1 \\
\hline & 2.Semi-rough grinding & 0.8 & 1 & 0.6 & 1 & 2 & 1.4 & 1.2 & 1 & 0.9 & 0.9 & 0.9 \\
\hline & 3.Semi-Finish plane & 0.6 & 0.5 & 1.2 & 1 & 0.5 & 1 & 1 & 1 & 1 & 1 & 1 \\
\hline & 4.NC milling & 3 & 3 & 2 & 1.5 & 0.8 & 0.5 & 0.8 & 0.8 & 1.5 & 1.5 & 1.5 \\
\hline \multirow{7}{*}{ Hole Processing } & 1.rough boring & 1 & 0.6 & 1 & 1 & 1 & 1 & 1 & 1 & 1 & 1 & 1 \\
\hline & 1.Semi-Finish boring & 1 & 0.6 & 1 & 1 & 1 & 1 & 1 & 1 & 1 & 1 & 1 \\
\hline & 2.rough-Finish grinding & 1.1 & 0.8 & 0.8 & 1 & 2 & 1 & 1 & 1 & 1.9 & 0.9 & 1 \\
\hline & 3.NC boring & 3 & 3 & 2 & 1.5 & 0.8 & 0.5 & 0.8 & 0.8 & 1.5 & 1.5 & 1 \\
\hline & 4.NC grinding & 3 & 0.8 & 2 & 1.5 & 1.6 & 0.8 & 0.8 & 1.5 & 1.2 & 1.2 & 1 \\
\hline & 1.Diamaond boring & 1.5 & 1 & 1.2 & 1 & 1 & 1 & 1 & 1 & 1.5 & 1.2 & 1 \\
\hline & 2.Ploishing & 0.1 & 0.8 & 0.1 & 1 & 3 & 1 & 1.4 & 1.4 & 2 & 1.2 & 1 \\
\hline
\end{tabular}

level and $2 \mu \mathrm{m}$. Ra0 axis. Evaluation list is available for systems ready to show some computational credit suggestions Table 2 .

As can be seen in Fig. 4, design of machine No. 2 and design of No. 2, machine 5 and design of No. 3, machine 9 and design of No. 4, machine 3 and design of No. 5, machine 8 is assigned. It is a way to minimize the cost. The genetic chart has an iterative but convergent and straight line diagram. The cuckoo chart executes the answer with precision and obtains the exact answer 4979 while the genetic chart has the final answer 6936.

\section{CONCLUSION}

In this study, a new optimization algorithm was proposed which was inspired by lifestyle of a bird called cuckoo. Special characteristics of cuckoos in egg laying and breeding had been the basic motivation for development of this new optimization algorithm for selecting a digital production machine design. Digital production is an important system for any manufacturer that needs to increase its competitiveness and profitability. The purpose of digital production is to create more efficient production processes and products. In this research, a new hybrid algorithm is proposed that combines COA-AHP to make ideal decision making of machining plan. As can be said in this problem, a scientific model of machine assembly and plan selection was presented.

\section{REFERENCES}

Blochliger, I., and N. Zufferey, 2008. A graph coloring heuristic using partial solutions and a reactive tabu scheme. Comput. Oper. Res., 35: 960-975.

Ge, F., Z. Wei, Y. Tian and Z. Huang, 2010. Chaotic ant swarm for graph coloring. Proceedings of the 2010 IEEE International Conference on Intelligent Computing and Intelligent Systems, October 29-31, 2010, IEEE, Xiamen, China, pp: 512-516.

Guan, X., Y. Wang and L. Tao, 2009. Machining scheme selection of digital manufacturing based on genetic algorithm and AHP. J. Intell. Manuf., 20: 661-669. 
Langdon, W., 1986. The Whale and the Reactor: A Search for Limits in an Age of High Technology. University of Chicago Press, Chicago, Illinois, USA., ISBN: 9780226902104, Pages: 200.

Lu, Z. and J. K. Hao, 2010. A memetic algorithm for graph coloring. Eur. J. Oper. Res., 203: 241-250.

Rajabioun, R., 2011. Cuckoo optimization algorithm. Applied Soft Comput., 11: 5508-5518.
Ray, B., A.J. Pal, D. Bhattacharyya and T.H. Kim, 2010. An efficient ga with multipoint guided mutation for graph coloring problems. Int. J. Signal Process. Image Process. Pattern Recognit., 3: 51-58.

Webster, F., 2002a. The Information Society Revisited. In: The Handbook of New Media, Lievrouwand, L.A. and S. Livingstone (Eds.). SAGE, London, England, UK., ISBN: 9780761965107, pp: 255-266.

Webster, F., 2002b. Theories of the Information Society. 2nd Edn., Routledge, New York, USA., ISBN-13: 9780415282000, Pages: 273. 